\title{
Panorama do uso de Laboratório Didático em Cursos de Ensino de Física, Modalidade a Distância, no País.
}

\author{
Maria Sônia Silva de Oliveira Veloso PPGECIM-ULBRA soniaufrr@gmail.com \\ Agostinho Serrano de Andrade Neto PPGECIM-ULBRA asandraden@gmail.com
}

\begin{abstract}
Resumo: Este artigo apresenta uma análise parcial sobre as características dos laboratórios didáticos para ministração das aulas experimentais em curso graduação de Física a distância no Brasil. Tem como objetivo realizar um diagnóstico desses laboratórios diante do que existe publicado em uma revisão da literatura da área. Por meio da pesquisa de campo, foram identificadas e analisadas as instituições que estão credenciadas na plataforma do eMEC. Foi elaborada uma tabela com os dados obtidos dos tipos de laboratórios e suas características. Como resultado, foram selecionadas 20 instituições que oferecem cursos de licenciatura a distância, assim como quatro tipos de laboratórios didáticos diversificados. Concluímos com uma análise destas instituições frente ao estado da arte da literatura em ensino de física, para a modalidade.
\end{abstract}

Palavras-chave: laboratório didático; ensino de Física; educação a distância; aulas experimentais.

\begin{abstract}
This paper presents a partial analysis of the characteristics of instructional laboratories for experimental classes courses of ongoing physics undergraduation on distance learning in Brazil. Aiming to make a diagnosis on the kind of laboratories that are used for the experimental classes in teaching physical distance in relation to which there is published in the literature. Through field research, the institutions that are accredited at the eMEC platform have been identified and analyzed. A table with the data acquired from laboatory types and their characteristics was constructed. As a result, were selected twenty institutions offering degree courses in the distance as well as four types of diversified teaching laboratories. We conclude with an analysis of those institutions as compared to what is reported in the state of the art literature for physics teaching in the modality of distance learning.
\end{abstract}

Keywords: laboratory - teaching; physics teaching; distance education; experimental classes.

\section{Introdução}

Como ensinar física e como o aluno aprende seus conceitos nas aulas experimentais na Educação a Distância - EaD, são algumas das principais preocupações de professores e pesquisadores voltados ao ensino de Física na atualidade. Embora tenham ocorrido muitos avanços nessa área, é evidenciado que ainda existe muito a estudar e a aprofundar, pois a EaD está evoluindo com a utilização da tecnologia, no caso, para inserção de laboratório didático com uma metodologia diversificada para ensinamento de experimentos.

O laboratório didático de Física tem um papel importante no ensino dessa área, principalmente por colocar os estudantes em contato com os fenômenos descritos por leis e teorias que permeiam a ciência. De acordo com Hodson (1994, p. 313), esse ambiente é propício a que os alunos testem suas hipóteses, indagações e curiosidades, e façam uso de sua criatividade, dessa forma transformando o laboratório didático em um ambiente 
em potencial para o desenvolvimento de uma cultura científica capaz de proporcionar aos envolvidos uma visão mais completa da Física.

Neste processo da evolução de modalidades de ensino não presencial, além das possibilidades de acesso ao conhecimento, por meio da utilização de tecnologias e metodologias para aulas experimentais no ensino de Física no País, é desenvolvida a oportunidade de verificar quais instituições oferecem cursos de licenciatura na modalidade de Educação a Distância - EaD, assim como de analisar as características dos laboratórios utilizados para oferecimento das aulas experimentais no curso de licenciatura em Física nessa modalidade, com a perspectiva de responder à seguinte pergunta: Qual o panorama do uso de laboratório didático em cursos de ensino de Física, modalidade a distância, no País, em comparação com estado da arte da literatura em ensino de Física?

Com o desenvolvimento desta pesquisa de campo, foram identificadas, no primeiro momento, as instituições que oferecem o curso de licenciatura em Física a distância, com o objetivo de apresentar um diagnóstico das características dos laboratórios utilizados para as aulas experimentais nessa modalidade de ensino no Brasil, diante do que existe publicado em uma revisão da literatura na área. Foi utilizado o sistema do $e M E C$, sendo verificadas as instituições que estão credenciadas na plataforma. Para tanto, foi delineado um panorama com os dados obtidos na análise dos tipos de laboratórios e suas características.

Com essa finalidade, este trabalho está dividido em cinco seções. A primeira é o marco referencial, apresentando alguns estudos realizados sobre laboratório didático para o ensino de Física em outros países, assim como uma subseção que aborda a reflexão sobre tipos de laboratórios. Na segunda seção, são apresentados a definição do problema e o método de pesquisa, com os procedimentos metodológicos utilizados. A terceira trata da análise e discussão dos resultados obtidos, fazendo um diagnóstico dos tipos de laboratórios, suas características e a identificação das instituições que oferecem curso de licenciatura em Física a distância. Na quarta seção, são comtempladas as considerações relativas a uma revisão da literatura e os resultados alcançados neste trabalho. Por fim, na última, está o embasamento das referências utilizadas.

\section{Marco Referencial}

Para discorrer sobre o laboratório didático e a utilização do mesmo para as aulas experimentais em curso de licenciatura em Física a distância, é proposta uma síntese de uma revisão literária de teóricos que destacam a importância do uso desse laboratório para essas aulas no ensino de Física e/ou de disciplina relacionada.

\subsection{Visão do Laboratório Didático no Ensino de Física}

O uso do laboratório didático na educação para o desenvolvimento de experimentos, especificamente para a área experimental em Física, constitui um elemento inovador e transformador no ensino e na aprendizagem do aluno, podendo interferir no fenômeno e no processo educacionais, sobretudo em contexto de educação a distância.

Um dos grandes desafios na implantação e no uso desses laboratórios é que realmente representem uma inovação nos métodos de ensino e uma melhoria dos processos de construção do conhecimento e da aprendizagem. Assim, "aprender fazendo" é uma expressão que se revela em atividades laboratoriais e se fortalece pela teoria construtivista, por considerar que o conhecimento deve ser construído pelo aprendiz, por meio da interação com o objeto, e não somente transmitido pelo professor (LINDSAY et 
al., 2007). É sustentado que, no laboratório, os estudantes desenvolvem habilidades sociais e de trabalho em equipe.

Nesse contexto Guillermo et al.(2013) destaca-se que o Laboratório Virtual, se bem conduzido, pode desenvolver habilidades sociais numa turma de uma disciplina e também realizar ações colaborativas em grupo. Nesse sentido, Martinez (1996) refere que diversos estudos realizados demonstram que os estudantes apoiados pela educação mediada com tecnologia necessitaram de um terço menos de tempo do que aqueles que utilizaram métodos tradicionais para superar etapas de ensino em laboratório real.

A pesquisa apresentada por Meisner et al. (2008) com o tema "Aprendendo a Física em um Ambiente Virtual: Existe Alguma?”, destaca que alunos não conseguem evidenciar a compreensão profunda de conteúdo em ciência quando submetidos ao processo convencional de instrução de palestras e demonstrações. Em seu trabalho, são mencionadas inúmeras pesquisas realizadas nos Estados Unidos sobre palestras cuidadosamente elaboradas, incluindo (passivo) visuais, seja in situ ou em um espaço virtual, as quais, porém, não ajudam a responder de forma afirmativa à pergunta feita por Hake (2007), "Educação a Distância e Aprendizagem na Sala de Aula: existe relação? ". Foi esse foco que possibilitou subsídios para elaborar o título deste trabalho, com a intenção de explicar essas tentativas de "experiências" educacionais, pretendendo verificar em que tipo de laboratório estão sendo trabalhadas as aulas experimentais e quais são as características dos laboratórios de Física nessa modalidade no Brasil.

Em sua pesquisa, (MEISNER et al., 2008) apresenta, sob o enfoque da abordagem de experimentação virtual, a utilização de laboratório didático, com o desenvolvimento da aprendizagem na autoria de física virtual assíncrona em ambientes laboratoriais. Dessa forma, foi criado o LabPhysics, que é um software modular e multipropósito podendo ser usado como uma plataforma para cursos, horizontalmente entre as ciências e verticalmente em uma disciplina específica. O primeiro curso - autoria com esse software foi um introdutório para faculdade, curso de Física em nível de mecânica. Percebeu-se que o LabPhysics inclui tanto o processo quanto o conteúdo da ciência, componentes essenciais a qualquer curso, para que os alunos possam iniciar o estudo da disciplina, assim como características para um laboratório didático.

No processo científico, esses pesquisadores destacam que exames laboratoriais detalhados e altamente interativos, com a tomada de decisão, a seleção de equipamentos e instrumentação, a coleta de dados, a análise e a capacidade de cometer erros, são essenciais componentes da experiência. O princípio orientador da implementação desse processo e o desenvolvimento tanto do software quanto da estrutura e a história de tutoriais que compreendem o curso "LabPhysics Mecânica” é a Oficina de Pedagogia de Modelagem, um aclamado programa financiado por uma empresa dos Estados Unidos que investiu nessa pesquisa para que os pesquisadores e os professores de Física pudessem verificar o funcionamento desse laboratório virtual.

Essa abordagem é um dos vários em movimento na reforma do ensino da Física e leva os alunos a investigar padrões na Física (ou realisticamente virtual!) mundial e mapeá-los em específicos sistemas conceituais utilizando inúmeras representações (MEISNER et al. 2008). Conforme Arruda et al. (2001), a estratégia de ensino de Física sob enfoque experimental é defendida por diversos investigadores e educadores do ensino de ciências, a partir de uma perspectiva de ensino cognitivista.

Nessa possibilidade de utilizar o laboratório didático virtual, enfatiza-se que a experimentação deve ser orientada segundo seu aspecto qualitativo, visando problematizar para obter uma situação de ensino mais significativa (VILLATORRE et al., 2008).Observa-se nesse trabalho de Meisner et al.(2008) que o LabPhysics é uma variação de modelos analisados de laboratórios didáticos em disciplinas experimentais de 
Física, no estudo para fins de modelagem.

Procurando verificar outras maneiras de utilizar laboratório didático, foi analisada uma pesquisa realizada na Universidade de Havana, em Cuba, por (LLERENA e GONZÁLEZ, 2010). O resultado sobre o uso de experimentos de Física simulados, que chamaram de "Simulações Virtuais de Experimentos de Professores" - (SVEP), é praticamente a única alternativa para experimentos de ensino. Em muitos casos, os professores sentiram que isso poderia substituir verdadeiros laboratórios tradicionais; no entanto, poucos estudos teóricos pedagogicamente fundamentados foram realizados para definir o verdadeiro alcance e as possibilidades educacionais dessas simulações.

Ao usar os processos de simulações, é de extrema importância que tanto o professor quanto o aluno estejam conscientes de que eles são um modelo simplificado da realidade, sob risco de assimilar uma ideia errada do fenômeno em estudo ( HECKLER et al., 2007).

Percebe-se que a pesquisa foi dividida em três grupos. O primeiro teve aulas no laboratório virtual com guias de explicações e aplicação dos simuladores. O segundo, no laboratório real, com instrumentos experimentais, sem contato com simuladores e o ambiente virtual. As aulas do terceiro grupo foram ministradas no laboratório virtual associado ao laboratório real.

Com a aplicação dessa pesquisa, foi concluído que o terceiro grupo apresentou maior possibilidade de aprender do que os outros dois, principalmente quando realizaram experimentos virtuais utilizando os simuladores. Hohenfeld e Penido (2009) falam sobre o que o uso da simulação pode oportunizar nas aulas experimentais:

\begin{abstract}
Nas Simulações, existe a possibilidade do teste das hipóteses uma vez que é possível mudar os parâmetros, ainda que isso seja difícil de acontecer em condições reais. De tal forma que permite a criação de hipóteses fora do modelo vigente, que podem ser testadas para alguns fenômenos, os quais não podem e ser observados com o aparato experimental disponível. (HOHENFELD e PENIDO, 2009).
\end{abstract}

Observa-se que, dessa forma, a perspectiva investigativa nas atividades experimentais pode ser estruturada a partir da complementaridade dos laboratórios tradicionais e virtuais. Trentin e Tauroco (2002) comprovaram por meio de uma pesquisa que um laboratório virtual tem que estar inserido em um ambiente de apoio. Esse ambiente é baseado em atividades que o aluno é convidado a visitar. Nelas há instruções e sugestões para que ele o tire o máximo proveito do ambiente, além do acesso a conteúdo complementares, como a utilização de experimentos virtuais com o complemento de simuladores, links de apoio, fórum, leituras, material didático, diário de bordo, chats, mural, bate- papo, perfil e testes on-line.

Silva (2009) destaca que, nos países do Reino Unido, na Austrália, nos Estados Unidos, em países da Europa e na Nova Zelândia, a EaD foi estudada de forma a viabilizar a testagem e o questionamento da metodologia que estava sendo empregada no ensino superior em cursos a distância. Foram verificadas melhorias principalmente na utilização de experimentos em laboratórios didáticos para o ensino de Física.

A possibilidade de o aluno aprender em um laboratório didático no ensino de Física, permite a interação de novos conhecimentos neste mundo globalizado.

Com o objetivo de comparar tipos de laboratórios, são discutidos, a seguir, alguns que podem ser utilizados para a ministração das aulas experimentais no ensino de Física.

\title{
2.2 Tipos de Laboratórios
}

Existem várias pesquisas relacionadas a tipos de laboratórios, entre as quais destacamos "Implementação de Laboratórios Virtuais em Realidade Aumentada para 
Educação a Distância”, de (FORTE et al. 2008). Esse artigo apresenta uma discussão sobre laboratórios virtuais implementados com multimídia, realidade virtual e realidade aumentada, analisando seus aspectos técnicos e educacionais e enfatizando a colaboração local e remota. Ressaltaremos nessa pesquisa exatamente os tipos de laboratórios e as vantagens e desvantagens. Os laboratórios são caracterizados, conforme os autores:

a) Laboratório Real - Ambiente físico com instrumentos para manuseios e guias de orientação nos momentos da montagem e de teste dos experimentos, por sua característica física, impede o acesso amplo a seus recursos. Mesmo quando se trata apenas de alunos de educação presencial, é preciso lembrar que o acesso a laboratórios reais também é dificultado, na medida em que estes são limitados fisicamente e via de regra não poderiam suportar todos os alunos que desejassem fazer seus experimentos nos momentos em que estão disponíveis, necessitando de agendamento para utilização. As vantagens são a interação com equipamentos reais, o trabalho colaborativo e os resultados reais.

b) Laboratório Virtual - Esses laboratórios distinguem-se bastante entre si e podem ser caracterizados principalmente de dois modos: por tipo de tecnologia empregada, sendo mais comuns a multimídia, aqueles em realidade virtual e os laboratórios em realidade aumentada, ou por aspectos de colaboração, os laboratórios que existem em ambientes de colaboração local e de colaboração remota.

Visto que o laboratório virtual se subdivide, Forte et al.(2008) fornece a seguinte explicação:

I) Virtual com multimídia - Podem apresentar sons, textos, animações, vídeos e imagens, a fim de que o conteúdo abordado seja apresentado de maneira ampla e com fácil compreensão. Os laboratórios multimídia podem ser distribuídos em CDs, como parte integrante do material didático de determinado curso, ou disponibilizados na internet para acesso on-line. Aqui também se introduzem experiências mais antigas de expansão do conhecimento laboratorial, feitas por meio de gravação, e disponibilização de conteúdo por vídeos ou DVDs, mostrando como é realizada determinada prática laboratorial. Como vantagens, fácil distribuição e baixo custo de desenvolvimento. Já como desvantagem, pouco contribui para o aprendizado das características práticas do ensino laboratorial.

II) Virtual com a realidade virtual - Caracteriza-se pela imersão total do usuário no mundo virtual, o que equivale dizer que ele é transferido para o ambiente programado por meio de recursos como óculos de visualização ou caves. Esses laboratórios trabalham com o conceito de simulação do ambiente laboratorial, podendo ser tão eficazes quanto sua representação fiel. Nas vantagens, alto índice de imersão e representação do ambiente real. E como desvantagens, alto custo de desenvolvimento e restrições de uso e distribuição porque precisam de hardwares específicos.

III) Virtual em realidade aumentada - Caracteriza-se pelo enriquecimento do mundo real mediante a adição de elementos virtuais. O usuário geralmente observa a ocorrência de eventos, a partir da tela do computador, sem precisar se munir de dispositivos especiais de visualização, e interage com os objetos virtuais com o auxílio de marcadores tangíveis, também sem necessitar de dispositivos especiais para a tarefa. Como vantagens, destacam-se o baixo custo de desenvolvimento, índice satisfatório de representação de aplicações, facilidade de interação e não haver necessidade de hardwares específicos. A desvantagem é o menor índice de imersão.

Nesses três modelos inseridos em laboratório virtual, percebe-se a evolução da tecnologia que Silva (2008) aborda, sendo cada uma de uma forma específica, mas com 
requisitos, conforme o autor destaca, de estimular o raciocínio do aluno para questionar, testar e buscar o resultado.

\section{Definição do Problema e Método de Pesquisa}

O objetivo desse trabalho é apresentar um diagnóstico sobre as características dos laboratórios utilizados para as aulas experimentais no ensino de Física a distância, quando comparados com o que é discutido no estado da arte da literatura em Ensino de Física no País. Nesse sentido, foi realizado o seguinte procedimento metodológico: pesquisa de campo, organizada em três momentos. No primeiro, foram verificadas as instituições que estão credenciadas na plataforma do $e M E C$, sendo realizado contato com essas instituições.

Em seguida, foi organizada uma tabela com as informações adquiridas no contato com as coordenações do curso de Física de cada instituição credenciada no sistema do eMEC. Essa tabela foi elaborada dando destaque ao que estava sendo investigado.

No procedimento do terceiro momento, foram pesquisados trabalhos relacionados ao tema que aqui está sendo abordado. Essa etapa foi caracterizada como uma análise de revisão.

Foi organizado um banco de dados de trabalhos relacionados sobre laboratório virtual no ensino de Física e características de laboratórios utilizados em cursos de licenciatura em Física ou disciplinas dessa área presentes nesses trabalhos. Para avaliação dos dados foi utilizado análise de revisão, a partir do qual foi focado em três categorias na busca de trabalhos publicados.

- Aulas experimentais e simulações;

- Laboratórios para cursos de Física ou afins de modalidade a distância;

- Tipos de laboratórios.

Essas categorias foram criadas após a análise dos trabalhos publicados que oferecem cursos de Física ou cursos afins na modalidade a distância no País. Dessa forma, foi concebida uma comparação das características dos laboratórios utilizados nesses cursos.

O trabalho é analisado e discutido com base nessas categorias e nas informações obtidas pelas instituições que estão credenciadas no eMEC.

\section{Análise e Discussão dos Resultados}

$\mathrm{Na}$ verificação dos tipos de laboratórios didáticos utilizados para curso de licenciatura em Física a distância e suas características, foram exploradas todas as instituições que estão credenciadas no sistema do eMEC. Obtiveram-se informações contidas no sistema, referentes ao ano de início do curso; contato do coordenador do curso; e regiões em que são ofertados. Desse modo, os dados foram organizados para que fossem realizadas as três etapas dessa pesquisa.

No primeiro momento, quando foram encaminhados os contatos com as instituições, observou-se que 20 estão credenciadas no sistema do eMEC, entre particular, federal e estadual, ofertando curso de licenciatura em Física a distância. Na segunda etapa, foi montada a Tabela 1, com as informações obtidas a partir desses contatos, listando as instituições, os tipos de laboratórios e observações.

Já no terceiro momento, foram efetuados estudos de caso que explicassem modelos de laboratórios didáticos na EaD e / ou sua utilização nas disciplinas envolvendo a Física. Foi elaborado um panorama de alguns países que já evidenciaram pesquisas relacionadas 
à evolução e utilização de tecnologia demonstrando o uso do laboratório didático para o desenvolvimento da aprendizagem no ensino a distância ou equivalente.

Percebeu-se também que o sistema do eMEC não tem informações completas, como o projeto pedagógico curricular, o telefone e o endereço eletrônico da coordenação do curso, fornecendo somente informações da reitoria, do site e o e-mail das instituições em geral. Por meio destes é que foram feitos os contatos, solicitando o telefone da coordenação do curso de Física a distância de cada instituição, para obtenção dos dados necessários à elaboração da tabela com as informações pertinentes.

Destaca-se, a seguir, a Tabela 1, com a identificação dos tipos de laboratórios, das instituições e observações caracterizando exatamente o laboratório que a instituição desenvolve e disponibiliza aos alunos. Os quatros laboratórios com nomes específicos foram propostos pelas instituições pesquisadas e pelas leituras realizadas no referencial teórico.

\subsection{Caracterizações de tipos de laboratórios encontrados nas instituições}

\section{pesquisadas}

Tabela 1- Caracterização dos laboratórios utilizados em cursos de licenciatura a distância no Brasil

\begin{tabular}{|c|c|c|}
\hline Tipos de Laboratórios & Observações & Instituição \\
\hline $\begin{array}{l}\text { 1. Laboratório } \\
\text { semipresencial }\end{array}$ & $\begin{array}{l}\text { - A instituição distribui o material } \\
\text { auto- explicativo para as aulas } \\
\text { experimentais no ambiente. } \\
\text { - Utilização de exemplos por meio } \\
\text { de simuladores e material didático. } \\
\text { - Utilização do laboratório real no } \\
\text { polo. } \\
\text { - Auxílio do tutor no polo presencial. }\end{array}$ & $\begin{array}{l}\text { UNIMES, UFC, } \\
\text { UFRN, UNEMAT, UFG, } \\
\text { UNIS, UFJF, } \\
\text { UFRPE, UFAL. }\end{array}$ \\
\hline $\begin{array}{l}\text { 2. Laboratório } \\
\text { virtual }\end{array}$ & $\begin{array}{l}\text { - A instituição distribui o material } \\
\text { didático para as aulas } \\
\text { experimentais no ambiente. } \\
\text { - Utilização de laboratório virtual no } \\
\text { ambiente. } \\
\text { - Utilização de simuladores no } \\
\text { ambiente. } \\
\text { Explicação sobre as aulas } \\
\text { experimentais pelo professor e } \\
\text { pelo tutor no ambiente. }\end{array}$ & Nenhuma Instituição \\
\hline $\begin{array}{l}\text { 3. Laboratório } \\
\text { presencial }\end{array}$ & $\begin{array}{l}\text { - A instituição distribui o material } \\
\text { didático para as aulas } \\
\text { experimentais no polo por meio } \\
\text { dos tutores. } \\
\text { Utilização do laboratório real no } \\
\text { polo. } \\
\text { As explicações ocorrem no } \\
\text { ambiente e no polo para o apoio } \\
\text { presencial. } \\
\text { As metodologias usadas nas aulas } \\
\text { experimentais para utilização de } \\
\text { material variam conforme o } \\
\text { professor e o tutor. } \\
\text { Não tem interação com laboratório } \\
\text { virtual. }\end{array}$ & $\begin{array}{l}\text { UNIFEI, UEM, } \\
\text { UFVJM, UFPC, } \\
\text { UECE,UFS. }\end{array}$ \\
\hline & $\begin{array}{l}\text { - A instituição entrega na residência } \\
\text { dos alunos kits e guias } \\
\text { explicativos. } \\
\text { - Utilização de material didático }\end{array}$ & \\
\hline
\end{tabular}




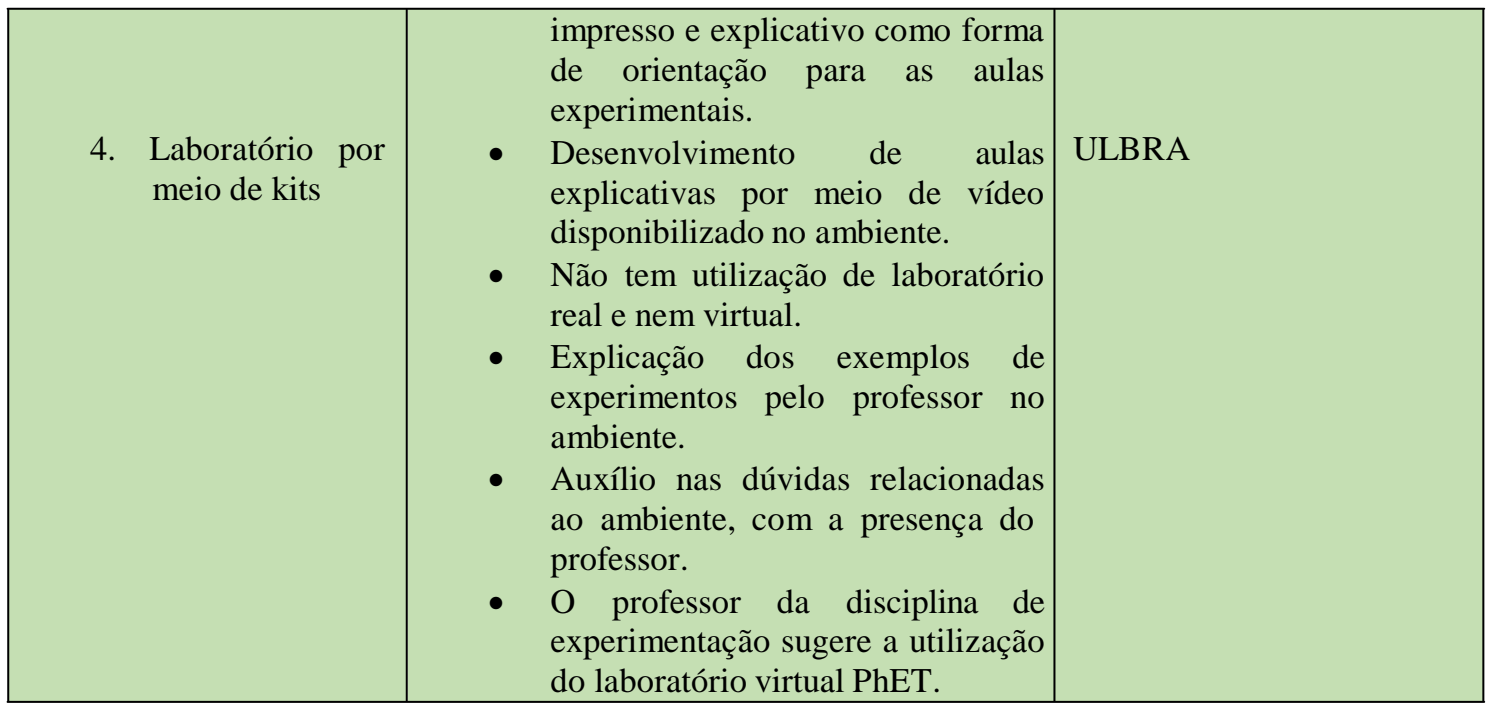

Na pesquisa realizada com as instituições e com o complemento da revisão de literatura, são destacados quatro tipos de laboratórios:

I. Laboratório semipresencial - é um laboratório em que os alunos fazem presença no ambiente real e também no virtual, tendo contato com experimentos reais e virtuais e leituras por meio de guias de aulas experimentais. Nota-se que, nesse tipo de laboratório, os alunos interagem com o tutor no polo presencial, havendo a possibilidade de tirar suas dúvidas presencialmente. Foi constatado que 11 instituições utilizam esse tipo de laboratório.

II. Laboratório virtual - é um laboratório totalmente virtual no ambiente de aprendizagem; porém, não foi observada a utilização desse modelo nas instituições credenciadas no eMEC. A caracterização desse modelo de laboratório foi baseada nas revisões literárias que foram apresentadas como comparação nesta pesquisa.

III. Laboratório presencial - é um laboratório com característica totalmente presencial, sendo disponibilizadas apenas as explicações no ambiente virtual, sem aplicação de atividades nesse ambiente. Nesse tipo de laboratório, o aluno precisa estar presente no polo para realização das atividades com o acompanhamento do tutor. Foi constatado que 8 instituições o utilizam.

IV. Laboratório por meio de kits - é um laboratório com característica totalmente a distância, porém orientado pelo ambiente, mas com utilização do laboratório virtual PhET. Dessa forma, o aluno tem que entrar no link sugerido pelo professor da disciplina. Nesse modelo, o aluno recebe um kit de acordo com o que será trabalhado na disciplina, acompanhado por um guia de instrução para montagem, realização de atividade e teste. Foi verificado que somente uma instituição utiliza esse modelo, conforme demonstrado na Tabela 1.

Na comparação das informações obtidas, notou-se que nenhuma instituição usa o modelo totalmente virtual, segundo observa Forte et al. (2008), relatando que esse tipo de laboratório pode ser subdividido em: laboratórios multimídia, laboratórios em realidade virtual e laboratórios em realidade aumentada, ou por aspectos de colaboração: ambientes de colaboração local e ambientes de colaboração remota.

\section{Considerações Finais}

Com a realização deste trabalho, consideramos que a evolução da $\mathrm{EaD}$ e a possibilidade de aprender em ambiente virtual vêm crescendo de modo satisfatório não 
só no Brasil, mas principalmente em outros países. Verificou-se que os países do Reino Unido, a Austrália, os Estados Unidos, países da Europa, a Nova Zelândia e Cuba, abordados no referencial teórico, se encontram bem adiantados tanto na evolução da EaD, quanto na tentativa de testar novas possibilidades de ideias na criação e utilização de softwares modular e multipropósito para cursos de ciências.

A relação de trabalhos investigados em outros países a respeito do tema se torna como base de comparação e também comprova que o Brasil necessita investir mais em pesquisas para o desenvolvimento de tecnologias que possam ser inseridas na EaD, principalmente porque foi destacado por este trabalho que outros países já há muito tempo vêm se preocupando em criar software de laboratório virtual para o funcionamento em disciplinas relacionadas à Física, favorecendo também todas as áreas da ciência.

Em relação aos trabalhos selecionados para comparação e suporte de conhecimento, houve um direcionamento ao verificar que as instituições que ofertam cursos de licenciatura em $\mathrm{EaD}$ no Brasil não necessariamente utilizam tecnologias sofisticadas abordadas no referencial teórico, como o LabPhysics, laboratórios multimídia, laboratórios em realidade virtual e em realidade aumentada. Foi comprovado, conforme demostrado na Tabela 1, na seção de resultados, que nenhuma instituição utiliza totalmente laboratórios virtuais.

Respondendo ao problema de pesquisa, ficou evidenciado que há diversos formatos de laboratórios didáticos inseridos na $\mathrm{EaD}$, desde cursos apoiados apenas em materiais impressos, kits de experimentos com guias para montar e testar, até aqueles que contam com recursos tecnológicos, porém com a utilização de simuladores. Entretanto, nenhum de ponta, como a criação de software para autoria de Física virtual assíncrona; ambientes laboratoriais, como o LabPhysics, destacado por Gerald et al. (2008).

Assim sendo, diante desta pesquisa, obtivemos não só as características dos laboratórios utilizados para aulas experimentais, mas também a possibilidade de verificar mais futuramente se há aprendizagem após as aulas que neles são ministradas. Espera-se que o resultado desta pesquisa possa contribuir como uma forma de reflexão para estimular professores a aprofundar seu interesse na aprendizagem em laboratório didático para o ensino de Física na EaD.

\section{Referências}

ARRUDA, S. M.; SILVA, M. R.; LABURÚ, C. E. Laboratório didático de física a partir de uma perspectiva kuhniana. Investigações em Ensino de Ciências, Porto Alegre, v. 6, n.1, 2001. Disponível em: http://www.if.ufrgs.br/public/ensino/revista.htm. Acesso em 12 de maio de 2014.

ANDRADE, J. A. N.; LOPES, N. C.; CARVALHO, W. L. P. Uma Análise Crítica do Laboratório Didático de Física: A Experimentação como uma ferramenta para a cultura científica. $\quad$ ENPEC 2009. $\quad$ Disponível em http://posgrad.fae.ufmg.br/posgrad/viienpec/pdfs/1161.pdf. Acesso em 10 de setembro de 2014.

COMARELLA, R. L.; RIBAS, J. C. C.; CATAPAN, A. H. O papel das mídias e o modelo de curso na educação a distância. RENOTE. Revista Novas Tecnologias na Educação, V. $8 \mathrm{~N}^{\circ} 1,2010$.

FORTE, C.; SANTIN, R.; OLIVEIRA, F. C.; KIRNER,C. Implementação de Laboratórios Virtuais em Realidade Aumentada para Educação à Distância, 2008. 
Disponível em http://www2.fc.unesp.br/wrva/artigos/50464.pdf. Acesso em 20 de setembro de 2014.

GUILLERMO, Oscar E. Patrón; Luiz. ENDRES, A. Magalhães; LIMA, José Valdeni. Laboratório Virtual de Aprendizagem Hidrolândia, 2013. Disponível em http://www.lume.ufrgs.br/bitstream/handle/10183/98671/Ensino2013_Resumo_32725.p df? sequence=1.Acessado em 10 de agosto de 2014 .

HAKE, R., Distance and Classroom Learning: Is There Any? ref 53 at. Disponível em: http://www.physics.indiana.edu/ hake. 2007. Acessado em 20 de outubro de 2014

HECKLER, V.; SARAIVA, M. F. O.; FILHO, K. S. O. Uso de simuladores, imagens e animações como ferramentas auxiliares no ensino/aprendizagem de óptica. Revista Brasileira de Ensino de Física, v. 29, n. 2, p. 267-273, 2007.

HODSON, D. Hacia un enfoque más crítico del trabajo de laboratório. Ensenãnza de las Ciencias, v.12, n.3, p. 299-313, 1994.

HOHENFELD, D. P.; PENIDO, M. C.. Laboratórios convencionais e virtuais no ensino de Física. VII Encontro Nacional de Pesquisa em Ensino de Ciências-Enpec, 2009.

LINDSAY, G., DOCKRELL, J. and STRAND, S., 2007, Longitudinalpatterns of behaviour problems in children with specific speech and language difficulties: child and contextual factors.British Journal of Educational Psychology,77,811 - 828.

LLERENA, D. Rodríguez; GONZÁLEZ, J. Llovera. Estudio comparativo de las potencialidades didácticas de las simulaciones virtuales y de los experimentos reales en la enseñanza de la Física General para estudiantes universitarios de ciências técnicas; 2010. Disponível em: http://www.lajpe.org/jan10/27_Llovera.pdf.

Acesso em 20 de agosto de 2014.

MATINEZ, Max Quiroz. La World Wide Web como poderosa herramienta didáctica em la education a distância. In: CONGRESSO IBERO-AMERICANO DE INFORMÁTICA,3., Barranquila. Actas. Barranquila, 1996. Disponível em: http://www.c5.cl/ieinvestiga/ribie96.htm. Acesso em 10 de outubro de 2014.

MEISNER, Gerald W.; HOFFMAN, H.; TURNER, M. Learning Physics in a Virtual Environment: Is There Any? Revista Latinoamericana de Física Educativa, v.2, n.2, p. 87-102, 2008.

SILVA, A. C.; Aprendizagem em Ambientes Virtuais e Educação a Distância. Editora Mediação, Porto Alegre, 2009.

TRENTIN, S. A. M.; TAROUCO, R. M. L. Proposta de Utilização de um Laboratório Virtual de Física na Melhoria do Processo de Ensino e Aprendizagem. Revista de Informática na Educação: teoria \& prática, v.5, n.2, (2002).

VILLATORRE, M. A.; HIGA, I.; TYCHANOWICZ, D. S., Metodologia do Ensino de Física. Curitiba: Editora Ibpex, 2008. 